\title{
Single-atom gating of quantum-state superpositions
}

\author{
CHRISTOPHER R. MOON ${ }^{1}$, CHRISTOPHER P. LUTZ² AND HARI C. MANOHARAN * \\ ${ }^{1}$ Department of Physics, Stanford University, Stanford, California 94305, USA \\ ${ }^{2}$ IBM Almaden Research Center, 650 Harry Road, San Jose, California 95120, USA \\ *e-mail: manoharan@stanford.edu
}

The ultimate miniaturization of electronic devices will probably require local and coherent control of single electronic wavefunctions. Wavefunctions exist within both physical real space and an abstract state space with a simple geometric interpretation: this state space-or Hilbert space-is spanned by mutually orthogonal state vectors corresponding to the quantized degrees of freedom of the real-space system. Measurement of superpositions is akin to accessing the direction of a vector in Hilbert space, determining an angle of rotation equivalent to quantum phase. Here, we show that an individual atom inside a designed quantum corral $^{1}$ can control this angle, producing arbitrary coherent superpositions of spatial quantum states. Using scanning tunnelling microscopy and nanostructures assembled atom-by-atom ${ }^{2}$, we demonstrate how single spins and quantum mirages ${ }^{3}$ can be harnessed to image the superposition of two electronic states. We also present a straightforward method to determine the atom path enacting phase rotations between any desired state vectors. A single atom thus becomes a real-space handle for an abstract Hilbert space, providing a simple technique for coherent quantum-state manipulation at the spatial limit of condensed matter.

Progress in quantum nanoscience has engendered a physically diverse array of controllable solid-state quantum systems ${ }^{4-6}$. The prototypical quantum system consists of two wavefunctions that can be coherently combined into superpositions. Here, we create and study superpositions of electron wavefunctions in nanoassembled quantum corrals where we can finely tune the geometry. These structures permit unique investigations of nanoscale electrons and their correlations, including information propagation $^{7}$, lifetime effects ${ }^{8}$, Kondo interactions ${ }^{9}$ and spinorbit coupling ${ }^{10}$. We use quantum corrals to take the traditional technique of gating, or the application of electrostatic potentials, to its smallest possible scale. Here, a single adatom couples only to a minute fraction of an electron's spatial extent. Rather than changing applied voltages, we make only geometric changes to the gate position, enabling adiabatic control of a two-state quantum system. Geometric and adiabatic manipulation of wavefunctions is a robust alternative to dynamic manipulation for various quantum technologies ${ }^{11,12}$.

We engineered an elliptical resonator to harbour degenerate wavefunctions whose superpositions could be manipulated. The solutions to the Schrödinger equation in a hard-walled ellipse possess two quantum numbers: $n$, the number of nodes crossing the minor axis, and $l$, half the number of nodal intersections along the perimeter (these map to the radial and angular momentum quantum numbers in a circle). By judiciously choosing an ellipse's deformation and size, two target wavefunctions can be made degenerate at any energy. We targeted states with even $n$ and nonzero $l$, which possess two widely separated regions of concentrated amplitude along the major axis. After analytically solving for the ellipse's eigenspectrum as a function of deformation (Fig. 1a), we aimed to design a corral with states $|n, l\rangle=|4,4\rangle$ and $|2,7\rangle$ degenerate precisely at the Fermi energy $E_{\mathrm{F}}$. These correspond to the 41st and 42nd most energetic states, or $\psi_{j} \equiv|j\rangle=|41\rangle$ and $|42\rangle$. $\mathrm{On} \mathrm{Cu}(111)$, where the surface state band edge is $0.445 \mathrm{eV}$ below $E_{\mathrm{F}}$ and $m^{*}=0.38$ bare electron masses ${ }^{13}$, this is theoretically achieved in a $2 a \times 2 b=157 \times 110 \AA$ elliptical resonator, whose full energy spectrum is shown in Fig. $1 \mathrm{~b}$.

We assembled our designed resonator using a home-built scanning tunnelling microscope operating in ultrahigh vacuum. The single-crystal $\mathrm{Cu}(111)$ substrate was prepared, cooled to $\sim 4 \mathrm{~K}$ and dosed with $\sim 15$ Co atoms per $(100 \AA)^{2}$. We individually manipulated $^{2} 44$ Co adatoms to bound the corral. With spectroscopy, we verified that modes $|41\rangle$ and $|42\rangle$ occurred within a few millivolts of each other (see Supplementary Information, Fig. S1). A constant-current (I) topograph of the finished structure is shown in Fig. 1c. To confirm that the wavefunctions $|\psi\rangle$ closely describe this system, we used them to calculate (see the Methods section) a theoretical topograph (Fig. 1d) that reproduces the data without any fitting parameters. Figure le shows the calculated contributions $c_{j}$ of the significant modes composing the topograph $z(\mathbf{r})$, such that $\left.z(\mathbf{r}) \propto \sum_{j} c_{j} \| \psi_{j}(\mathbf{r})\right\rangle\left.\right|^{2}$.

Next, we added a nanoscopic gate: a single cobalt atom. While moving the adatom across the ellipse-effectively sweeping a local electrostatic potential across the eigenstates-we measured topographs (Fig. 2a-d) and simultaneously acquired $\mathrm{d} I / \mathrm{d} V$ image maps. By subtracting the $\mathrm{d} I / \mathrm{d} V$ map of the empty ellipse, we created $\mathrm{d} I / \mathrm{d} V$ difference maps (Fig. $2 \mathrm{e}-\mathrm{h}$ ). We began by placing the gate atom at one of the maxima of the calculated $|2,7\rangle$ state. The resultant difference map (Fig. 2e) strongly resembles the $|2,7\rangle$ state. Surprisingly, however, when the Co atom was moved rightward to one of the strong maxima of the state $|4,4\rangle$, the image produced (Fig. $2 \mathrm{~g}$ ) was manifestly different from either of the two eigenstates.

We will show that our $\Delta \mathrm{d} I / \mathrm{d} V$ maps are images of superpositions: phase-coherent $\left.\left|\sum_{j} a_{j}\right| \psi_{j}\right\rangle\left.\right|^{2}$. This is in contrast to typical scanning tunnelling microscope measurements, such as the topograph above, where the sum of tunnelling through independent channels ${ }^{14}$ yields signals proportional to phaseinsensitive $\left.\sum_{j} c_{j} \| \psi_{j}\right\rangle\left.\right|^{2}$. To demonstrate this result, we reproduce 
a

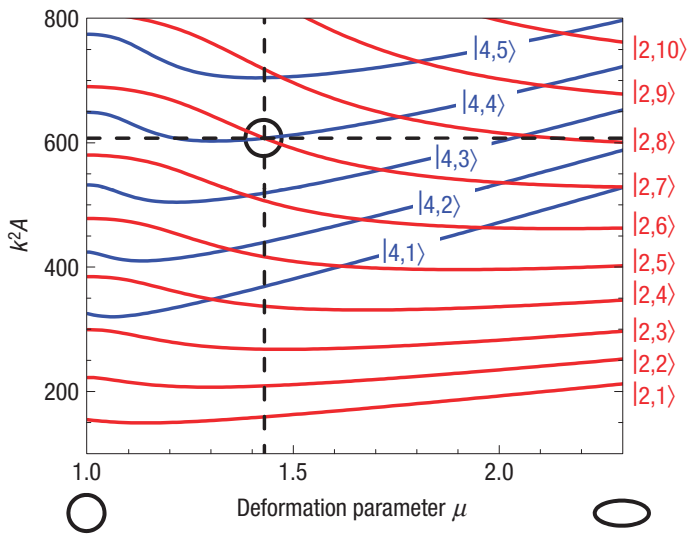

G

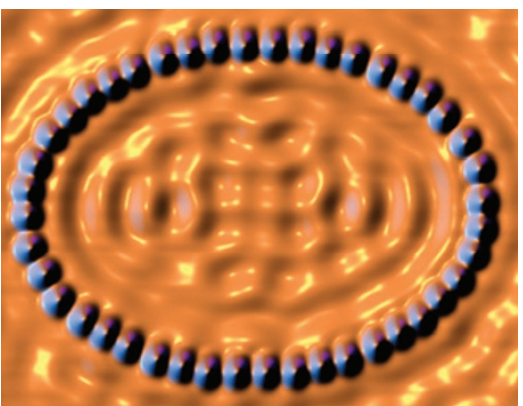

b

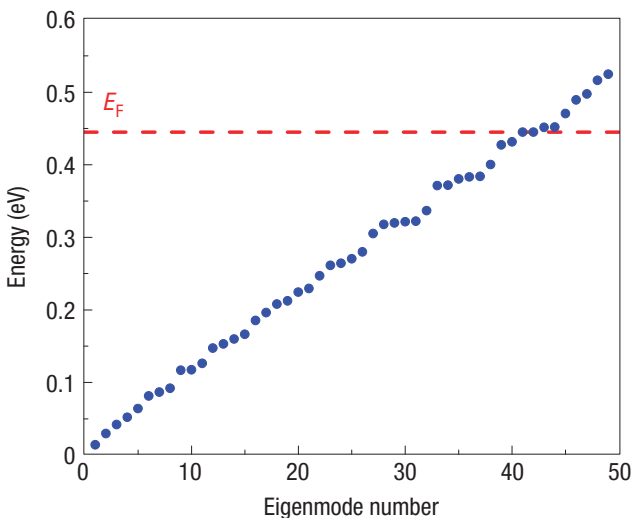

d

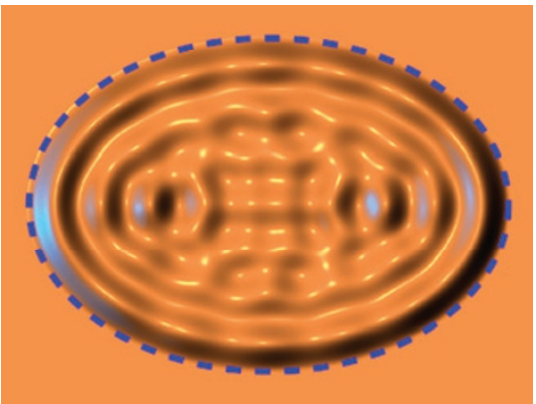

e

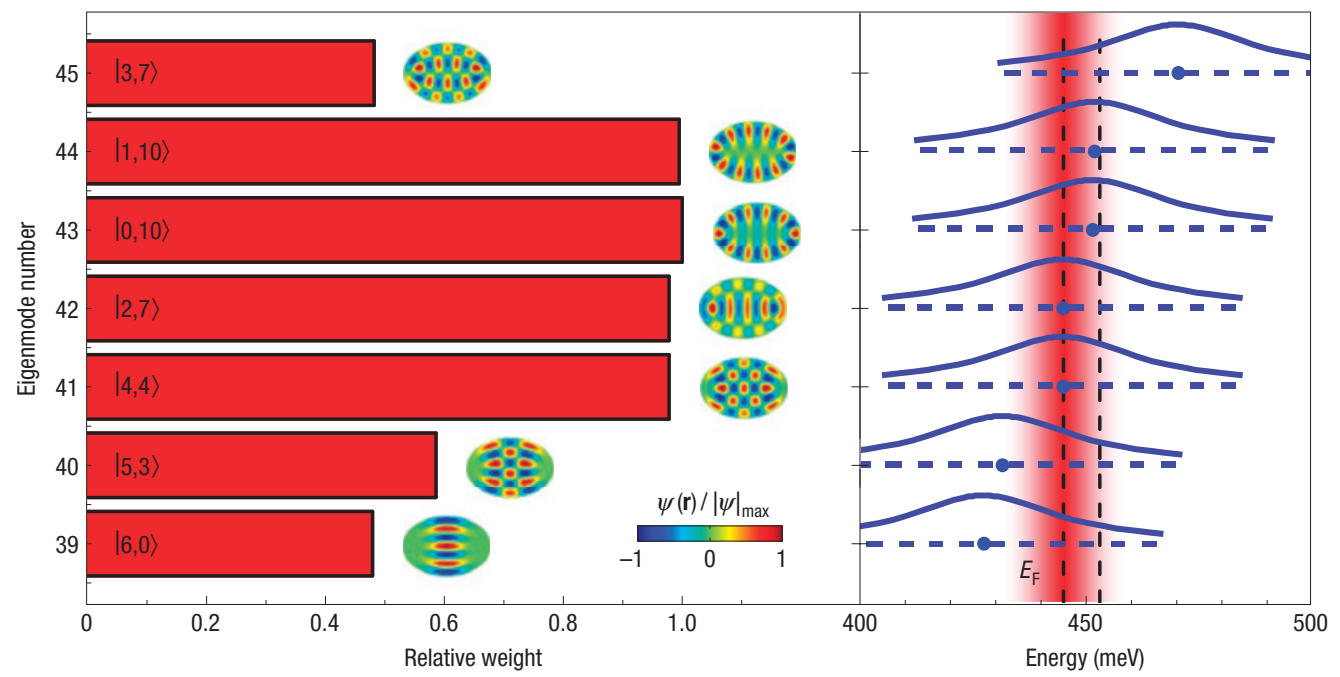

Figure 1 Designed degeneracy in a quantum corral. a, Eigenspectrum of desirable 'double-peaked' modes of the elliptical box calculated analytically as a function of its deformation parameter $\mu=a / b$, where $a$ and $b$ are the semi-major and semi-minor axes of the ellipse, respectively. The spectrum is shown in terms of the dimensionless energy parameter $k^{2} A$, where $A$ is the area of the ellipse and $k=\sqrt{2 m^{*} E / \hbar^{2}}$ is the wavenumber of an electron with effective mass $m^{*}$ and energy $E$. Intersecting dashed lines mark the parameters for our engineered degeneracy. $\mathbf{b}$, Energy spectrum for a $\mu=1.43$ elliptical resonator with $a=79.2 \AA$ (corresponding to circled degeneracy in $\mathbf{a}$ ). c, $175 \times 135 \AA$ constant- $/$ topograph $(V=8 \mathrm{mV}, I=1 \mathrm{nA})$ of the actual corral constructed. d, Simulated topograph calculated from the eigenmodes using a Green's function method. e, Left: relative weights of each squared wavefunction composing the topograph in $\mathbf{d}$, with corresponding $\psi(\mathbf{r})$ maps; right: energies of the seven modes in the corral lying closest to $E_{\mathrm{F}}$. Schematic lorentzians indicate the $40 \mathrm{meV}$ observed linewidth of the states in $\mathrm{d} / / \mathrm{d} V$ spectra. Vertical dashed lines mark the energy window for tunnelling electrons in our measurements. The Kondo resonance at $E_{\mathrm{F}}$ with width $T_{\mathrm{K}}=53 \mathrm{~K}$ is shown shaded in red.

the difference maps as linear combinations of the states $|\psi\rangle$ of the unperturbed elliptical corral. These coherent superpositions (Fig. 2i-1) are an excellent match to the mirage data. Any methods neglecting phase interference cannot reproduce our observations (see Supplementary Information, Fig. S2).

Electrons in quantum corrals are well modelled by particle-ina-box solutions to the Schrödinger equation because the surface state wavelength $\left(30 \AA\right.$ in $\mathrm{Cu}(111)$ at $\left.E_{\mathrm{F}}\right)$ is much larger than the spacing between the wall atoms $s^{1,3,10,15-18}$. As a first clue to the underlying physics, the original report of the quantum mirage ${ }^{3}$ pointed out the similarity between the solitary eigenfunction closest to $E_{\mathrm{F}}$ and the spatial fine structure around the projected Kondo image. A complementary approach ${ }^{10,19-24}$ treats the wall atoms as discrete scatterers of electron waves and solves the quantum 




b

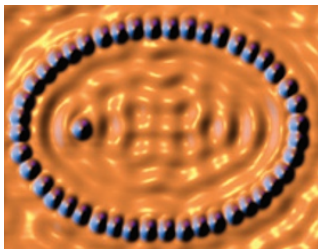

c

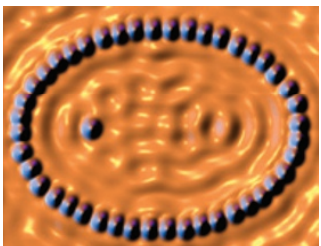

d

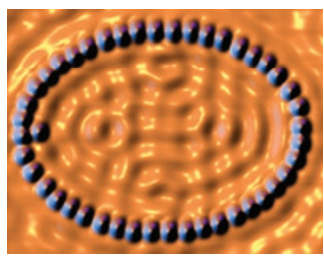

$50 \AA$
Q

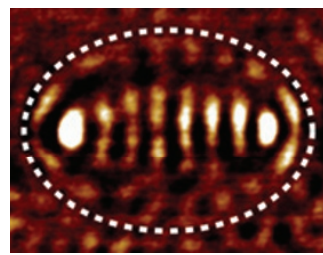

f

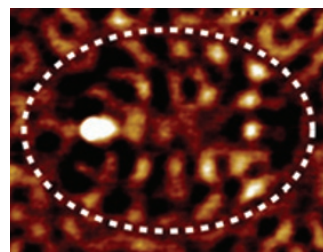

(])

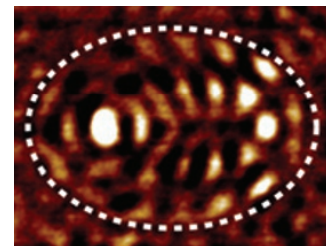

h

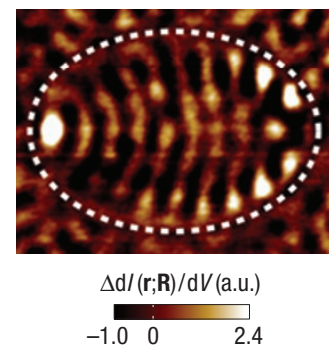

i

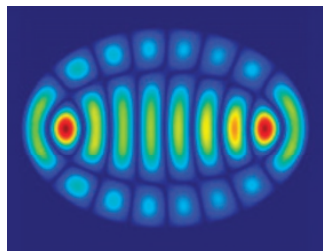

J

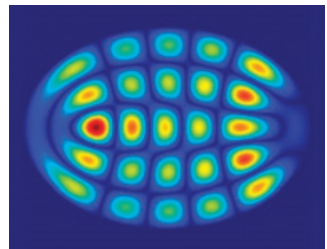

k

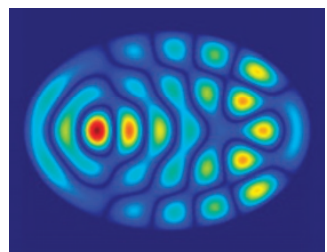

I
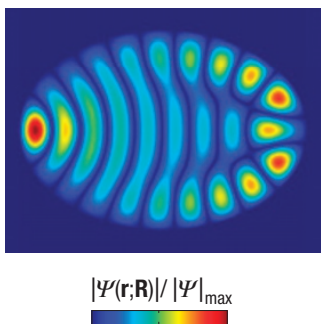

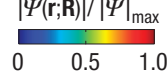

m



I

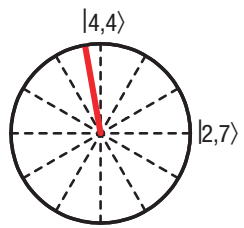

0

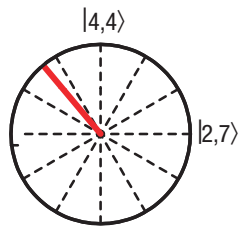

p

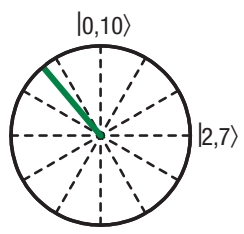

$\theta(\mathbf{R})$

Figure 2 Single-atom gating and read-out of quantum-state superpositions. a-d, Constant- $/$ topographs $(V=8 \mathrm{mV}, I=1 \mathrm{nA})$ of the elliptical electron resonator containing a single control atom at four different locations R. e-h, Simultaneously acquired d $l / d V$ difference maps $\left(V_{\mathrm{ac}}=2 \mathrm{mV}\right.$ r.m.s. at $\left.1,007 \mathrm{~Hz}\right)$ manifesting the quantum mirage. A dotted line traces the locations of the wall atoms that have cancelled out. i-I, Each mirage reveals a specific superposition $\Psi(\mathbf{R})$ of two empty-corral eigenmodes that we control with the atomic gate. $\mathbf{m}-\mathbf{p}$, The internal atom thus acts as a handle for a single quantum phase angle in a two-dimensional Hilbert space. These extracted phase angles are used to construct the superpositions shown in $\mathbf{i}-\mathbf{I}$.

multiple-scattering problem. In fact, the scattering theory was also applied $^{20}$ to produce very compelling computed matches to the mirage data and extract the Kondo phase shift. In this previous experimental and theoretical work, the structures studied had modes separated in energy by more than their linewidths- the state manifold was essentially non-degenerate. Here, we focus on the engineered degenerate case where two or more states are forced to coherently superpose by the added gate atom.

Although the exact origin of the Kondo resonance in our system is still debated ${ }^{25,26}$, microscopic calculations have indicated that the many-body complexity is manifested in the energy dependence of the density of states, whereas its spatial dependence can be understood from a single-particle perspective ${ }^{16,18}$. Regardless of its origin, we have verified that Kondo scattering calculations ${ }^{21}$ can reproduce many of the details of our images. However, the simplest and most intuitive model that reproduces the data requires only two electron wavefunctions and their elementary superpositions. Indeed, the success of our analysis is perhaps surprising in light of the well-known softness of the corral walls ${ }^{19}$. Without negating more complex approaches, this work vindicates the simple eigenmode picture of quantum corrals, which is relevant and useful to a variety of emerging applications.

For each superposition in Fig. 2, we selected the coefficients $a_{j}$ to create $\Psi(\mathbf{r} ; \mathbf{R})=\sum_{j} a_{j}(\mathbf{R})\left|\psi_{j}(\mathbf{r})\right\rangle$, where $\mathbf{R}$ is the atom position and $\mathbf{r}$ is a position within the ellipse, subject to the normalization constraint $\sum_{j}\left|a_{j}\right|^{2}=1$. If only two wavefunctions participate for a given $\mathbf{R}$, the state can be represented by a vector on a Bloch sphere: $|\Psi\rangle=\cos (\theta)\left|\psi_{1}\right\rangle+\mathrm{e}^{i \phi} \sin (\theta)\left|\psi_{2}\right\rangle$. However, timereversal symmetry requires that $|\Psi\rangle$ be real, so we are restricted to $\phi \in\{0, \pi\}$. Thus, we describe the mirage-projecting superposition with a single phase angle $\theta$, using $|\Psi\rangle=\cos (\theta)\left|\psi_{1}\right\rangle+\sin (\theta)\left|\psi_{2}\right\rangle$. Supplementary Information, Video S1 shows how $|\Psi\rangle$ evolves as the phase angle is varied in the space composed of $|2,7\rangle$ and $|4,4\rangle$. The angles used to create the superpositions for each gate atom location are indicated in Fig. $2 \mathrm{~m}-\mathrm{p}$. This decomposition demonstrates that by translating the atom in real space, we are effecting a rotation in the Hilbert space spanned by the two unperturbed states. For example, nudging the atom back by $5 \AA$ from the position in Fig. $2 c$ to $b$ achieves a state-space rotation of $30^{\circ}$.

The full Hilbert space of unperturbed wavefunctions is richer than the 2-space sampled in the first three rows of Fig. 2. Seven modes- $|39\rangle$ to $|45\rangle$, the set shown in Fig. 1e-significantly overlap both the energy range of our tunnelling electrons and the energies where Kondo processes can occur (governed by the Kondo temperature $T_{\mathrm{K}}$ ). However, at a given position $\mathbf{R}$, most of these modes have little amplitude and can be disregarded. For example, the atom positions in Fig. $2 \mathrm{a}-\mathrm{c}$ lie within a subspace of $\mathbf{R}$ where only states $|41\rangle$ and $|42\rangle(|4,4\rangle$ and $|2,7\rangle)$ have any significant presence. When the gate atom is moved to the extreme side of the ellipse 

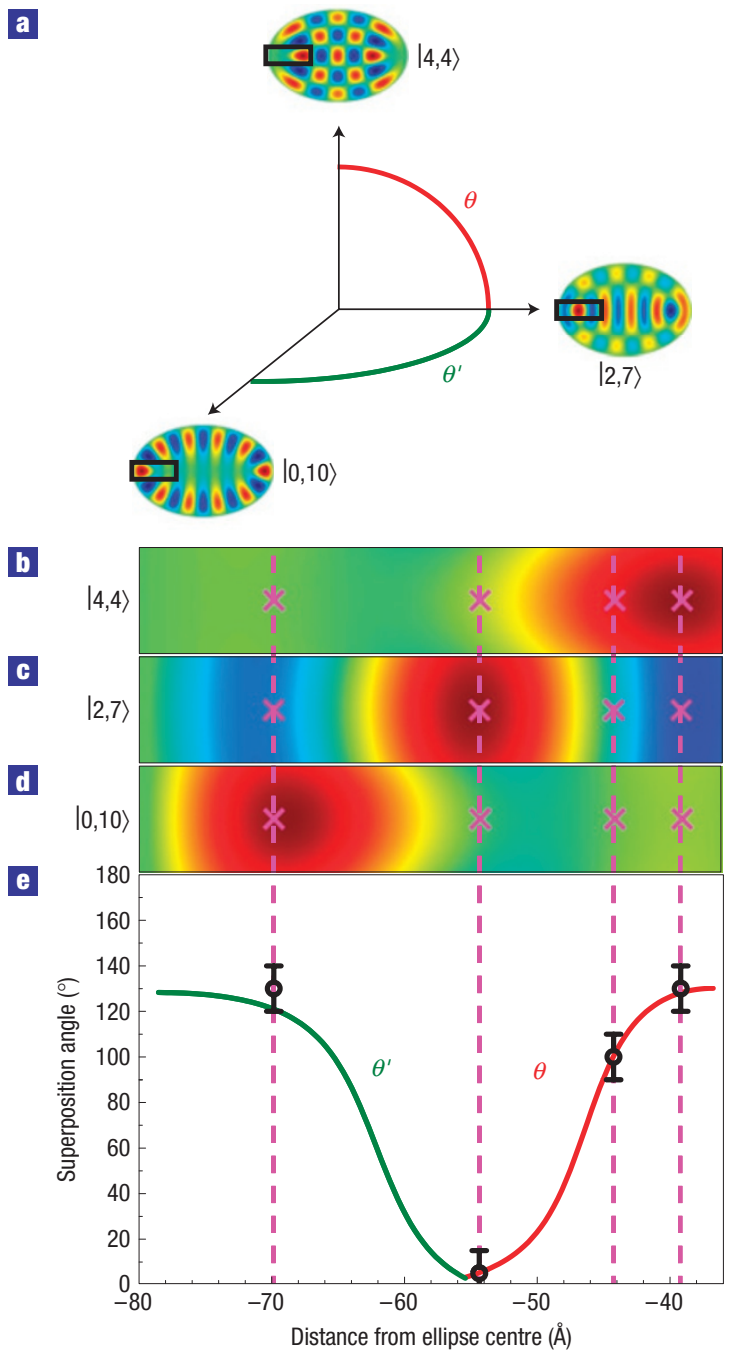

Figure 3 Complete indexing of two-dimensional Hilbert spaces. a, Schematic diagram of the two Hilbert space angles $\theta$ and $\theta^{\prime}$. b-d, Close-up views (see black rectangles in a) of the three eigenstates superposed in Fig. 2: $|4,4\rangle,|2,7\rangle$ and $|0,10\rangle$ (states $|41\rangle,|42\rangle$ and $|43\rangle$ respectively). The atom positions R in Fig. 2 are marked by crosses. e, Calculated Hilbert space phase angle as the control atom is translated along the major axis of the ellipse. Data points are the phase angles measured from Fig. $2 m-p$. The error bars indicate the accuracy limit of the fits of the difference maps (Fig. 2e-h) to the theoretical superpositions (Fig. 2i-l). Wavefunction amplitudes plotted with same colour scale as in Fig. 1e.

(Fig. 2, fourth row), $|41\rangle$ no longer contributes but is replaced by a 'whispering gallery' mode, $|43\rangle(|0,10\rangle)$. Here, an atom gates superpositions of $|42\rangle$ and $|43\rangle$, defining a new Hilbert 2-space and a new angle $\theta^{\prime}$. The dual Hilbert spaces (Fig. 3a) comprise an overall three-dimensional space; the crossover between planes in this space can be inferred from the structure of the wavefunctions (Fig. 3b-d).

To generate arbitrary superpositions, we require a way to predict the mirage-projecting wavefunction for any atom position $\mathbf{R}$. We accurately reproduced our data using degenerate perturbation theory, representing the gate atom with a delta function potential: $V=\alpha \delta(\mathbf{r}-\mathbf{R})$. These perturbation theory results are implicit in microscopic Green's function results ${ }^{16,18}$ in our engineered limit that the separation between relevant corral wavefunctions is much less than their linewidth. Physically, a single

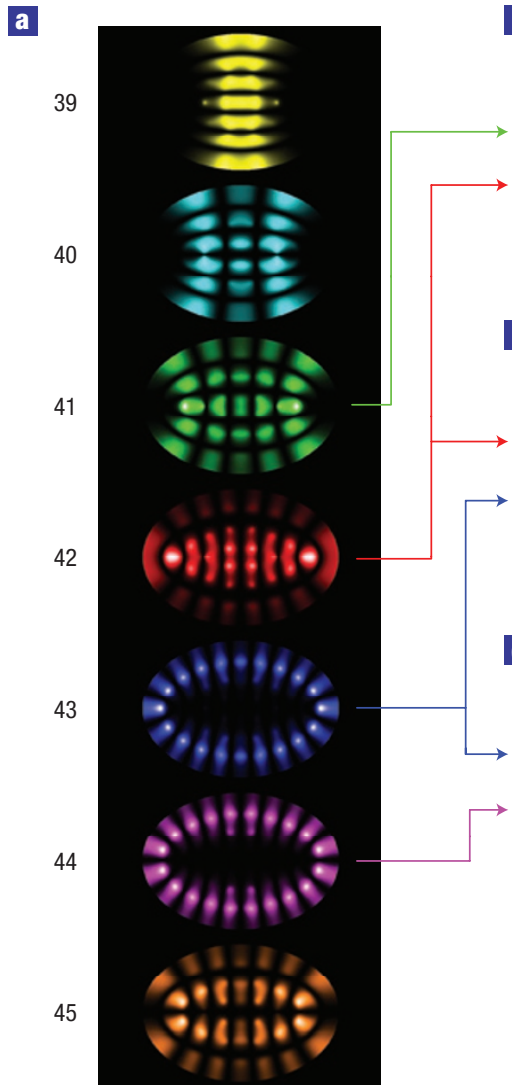

b

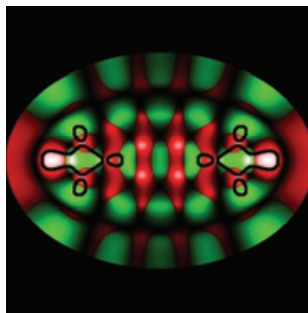

G

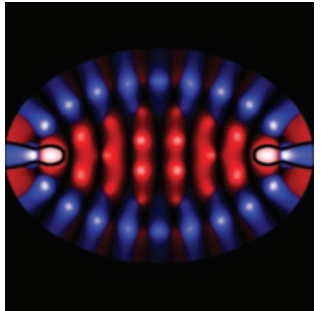

d
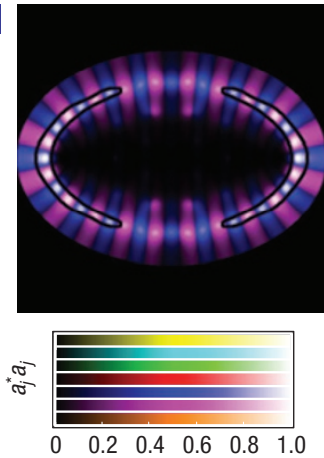

Figure 4 Superposition coefficient maps. a, Squared superposition coefficients $a_{j}^{*} a_{j}$ of each numbered eigenmode $j$ as a function of atom location $\mathbf{R}$. A white point in an eigenmode's a map corresponds to an adatom position that projects a quantum mirage composed purely of that state; a black point means the state will not be observed. $\mathbf{b}-\mathbf{d}$, Examples of subsets of $\mathbf{R}$ indexing two-dimensional Hilbert spaces: $|41\rangle \leftrightarrow|42\rangle,|42\rangle \leftrightarrow|43\rangle$ and $|43\rangle \leftrightarrow|44\rangle$. The a maps for two modes are superimposed as intersecting surfaces. Black lines delimit areas of the ellipse where no other modes have significant amplitude; a gate atom within these areas fully indexes a Hilbert space describable by a single phase angle $\theta$.

atom placed inside the corral causes the degenerate wavefunctions to reorganize themselves into new zeroth-order wavefunctions. One is the superposition having the greatest amplitude possible at the location of the adatom and projects the mirage; this state is 'tagged' and imaged by spectral mapping. All other combinations possess a node at $\mathbf{r}=\mathbf{R}$ and are therefore insensitive to the perturbation and ignored by our differential measurements. The energy shift of the perturbed mode is proportional to $\alpha$; we detect a shift of a few millielectrons at most, far less than the width of the states, so that this wavefunction remains energetically degenerate. Theoretical and experimental results for the Hilbert space angle while translating the gate atom across the two adjacent 2-spaces are shown in Fig. 3e; their excellent agreement demonstrates the validity of this analysis. Remarkably, for both spaces, all physically distinguishable combinations of the basis states can be created by the atom, limited only by the discreteness of adatom positions allowed by the substrate lattice.

The superpositions for atoms in regions where more than two wavefunctions have significant amplitude cannot be described by a single angle and are more difficult to visualize. In general, the state vector composed of the coefficients of the seven degenerate 
$|\psi(\mathbf{r})\rangle$ lies on the unit 7-sphere. Figure 4a shows ' $a$ maps', which graphically show the theoretical superposition coefficients produced by an atom at each point in the ellipse. The $j$ th map is a plot of $\left|a_{j}(\mathbf{R})\right|^{2}$. An atom at a white point in the $j$ th map will generate a quantum state that is purely $|\Psi(\mathbf{r})\rangle=\left|\psi_{j}(\mathbf{r})\right\rangle$. Sliding that atom to an adjacent white spot in the $k$ th map along a path where no other states contravene will continuously rotate the state vector from $\left|\psi_{j}(\mathbf{r})\right\rangle$ to $\left|\psi_{k}(\mathbf{r})\right\rangle$.

We find that almost all two-dimensional subspaces of the full seven-dimensional manifold can be fully indexed by a single atom. Figure $4 \mathrm{~b}-\mathrm{d}$ shows three examples of subspaces of $\mathbf{R}$ that index two-dimensional Hilbert spaces. The subspaces in Fig. 4b,c encompass the experimental points shown in Fig. 3; Supplementary Information, Video S2 shows theoretical results for atom paths that further explore these spaces. In each example, we plot the two $a$ maps as intersecting surfaces. Black lines demarcate the sets of $\mathbf{R}$ where $a_{1}^{2}+a_{2}^{2}>0.8$ for all of the set and $\left\{\left|a_{1}\right|,\left|a_{2}\right|\right\}>0.1$ for some point in each set-that is, where two and only two states are observable. This demonstrates a simple means to collapse a multidimensional Hilbert space onto a two-dimensional subspace, within which a state vector can be rotated using real-space atomic gate manipulation.

Our methods have direct applicability to other lowerdimensional nanostructures. The potential imposed on the confined states of our quantum corral by the control atom is analogous to any sufficiently local and movable gate potential. Selective gating of quantum superpositions in systems such as semiconductor ${ }^{27-29}$ quantum dots should be readily achievable with scanning or otherwise mobile gates. The atom-gating method we have used also has analogies in cavity perturbation techniques in microwave resonators ${ }^{30,31}$. As quantum two-state systems can in general be mapped to a pseudospin, here the real-space atom position maps to the direction of an effective magnetic field that couples to this spinor. We anticipate that atomic gating will enable detection and manipulation of other fundamental phases, including the Berry and Aharonov-Bohm phases, by using atom paths traversing properly tuned quantum manifolds.

\section{METHODS}

We used particle-in-an-elliptic-box wavefunctions $\psi_{j}$ to calculate the local density of states at position $\mathbf{r}$ and energy $\epsilon$,

$$
\operatorname{LDOS}(\mathbf{r}, \epsilon)=-\frac{1}{\pi} \operatorname{Im}\left(\sum_{j} \frac{\left|\psi_{j}(\mathbf{r})\right|^{2}}{\epsilon-\epsilon_{j}+i \delta_{j}}\right),
$$

which we numerically integrated to recreate the expected topograph height ${ }^{14}$,

$$
z(\mathbf{r}) \propto \int_{E_{\mathrm{F}}}^{E_{\mathrm{F}}+V} \operatorname{LDOS}(\mathbf{r}, \epsilon) \mathrm{d} \epsilon,
$$

at the experimental sample bias of $V=8 \mathrm{meV}$.

The linewidth of the electron states has been alternately ascribed to tunnelling across the corral barrier ${ }^{18,24}$ or coupling to the bulk states through inelastic scattering at the walls ${ }^{21}$ and intrinsic lifetime effects (for example, electron-phonon interactions $)^{32}$. Regardless of its origin, the broadening can be accounted for by a phenomenological lifetime added to particle-in-a-box wavefunctions. We used an electron self-energy $\delta=\Gamma / 2=20 \mathrm{meV}$ for all states, as per the $40 \mathrm{meV}$ linewidth $\Gamma$ observed in $\mathrm{d} I / \mathrm{d} V$ spectra for modes close to
$E_{\mathrm{F}}$. As contributions from these modes dominate the density of states relevant to our experiments, the variation of $\delta$ for modes far from $E_{\mathrm{F}}$ does not affect our calculation.

\section{Received 13 November 2007; accepted 29 February 2008; published 6 April 2008.}

\section{References}

1. Crommie, M. F., Lutz, C. P. \& Eigler, D. M. Confinement of electrons to quantum corrals on a metal surface. Science 262, 218-220 (1993).

2. Stroscio, J. A. \& Eigler, D. M. Atomic and molecular manipulation with the scanning tunneling microscope. Science 254, 1319-1326 (1991).

3. Manoharan, H. C., Lutz, C. P. \& Eigler, D. M. Quantum mirages formed by coherent projection of electronic structure. Nature 403, 512-515 (2000).

4. Kouwenhoven, L. \& Marcus, C. Quantum dots. Phys. World 11, 35-39 (1998)

5. Tans, S. J. et al. Individual single-wall carbon nanotubes as quantum wires. Nature 386, 474-477 (1997).

6. Woodside, M. T. \& McEuen, P. L. Scanned probe imaging of single-electron charge states in nanotube quantum dots. Science 296, 1098-1101 (2002).

7. Eigler, D. M. et al. Information transport and computation in nanometre-scale structures. Phil. Trans. R. Soc. Lond. A 362, 1135-1147 (2004).

8. Braun, K. F. \& Rieder, K. H. Engineering electronic lifetimes in artificial atomic structures. Phys. Rev. Lett. 88, 096801 (2002).

9. Rossi, E. \& Morr, D. K. Spatially dependent Kondo effect in quantum corrals. Phys. Rev. Lett. 97, 236602-236604 (2006)

10. Walls, J. D. \& Heller, E. J. Spin-orbit coupling induced interference in quantum corrals. Nano Lett. 7, 3377-3382 (2007).

11. Duan, L. M., Cirac, J. I. \& Zoller, P. Geometric manipulation of trapped ions for quantum computation. Science 292, 1695-1697 (2001).

12. Zanardi, P. \& Lloyd, S. Topological protection and quantum noiseless subsystems. Phys. Rev. Lett. 90, 067902 (2003).

13. Crommie, M. F., Lutz, C. P. \& Eigler, D. M. Imaging standing waves in a two-dimensional electron gas. Nature 363, 524-527 (1993).

14. Tersoff, J. \& Hamann, D. R. Theory of the scanning tunneling microscope. Phys. Rev. B 31, 805-813 (1985).

15. Aligia, A. A. Many-body theory of the quantum mirage. Phys. Rev. B 64, 121102 (2001).

16. Porras, D., Fernández-Rossier, J. \& Tejedor, C. Microscopic theory for quantum mirages in quantum corrals. Phys. Rev. B 63, 155406 (2001)

17. Schmid, M. \& Kampf, A. P. Mirages, anti-mirages, and further surprises in quantum corrals with non-magnetic impurities. Ann. Phys. (Leipzig) 12, 463-470 (2003).

18. Aligia, A. A. \& Lobos, A. M. Mirages and many-body effects in quantum corrals. J. Phys. Condens. Matter 17, S1095-S1122 (2005).

19. Heller, E. J., Crommie, M. F., Lutz, C. P. \& Eigler, D. M. Scattering and absorption of surface electron waves in quantum corrals. Nature 369, 464-466 (1994).

20. Fiete, G. A. et al. Scattering theory of Kondo mirages and observation of single Kondo atom phase shift. Phys. Rev. Lett. 86, 2392-2395 (2001).

21. Fiete, G. A. \& Heller, E. J. Colloquium: Theory of quantum corrals and quantum mirages. Rev. Mod. Phys. 75, 933-948 (2003).

22. Agam, O. \& Schiller, A. Projecting the Kondo effect: Theory of the quantum mirage. Phys. Rev. Lett. 86, 484 (2001).

23. Correa, A., Hallberg, K. \& Balseiro, C. A. Mirages and enhanced magnetic interactions in quantum corrals. Europhys. Lett. 58, 899 (2002).

24. Rahachou, A. I. \& Zozoulenko, I. V. Elastic scattering of surface electron waves in quantum corrals: Importance of the shape of the adatom potential. Phys. Rev. B 70, 233409 (2004).

25. Knorr, N., Schneider, M. A., Diekhoner, L., Wahl, P. \& Kern, K. Kondo effect of single Co adatoms on Cu surfaces. Phys. Rev. Lett. 88, 096804 (2002).

26. Lin, C. Y., Castro Neto, A. H. \& Jones, B. A. Microscopic theory of the single impurity surface Kondo resonance. Phys. Rev. B 71, 35417 (2005).

27. Eriksson, M. A. et al. Cryogenic scanning probe characterization of semiconductor nanostructures. Appl. Phys. Lett. 69, 671-673 (1996).

28. Topinka, M. A. et al. Imaging coherent electron flow from a quantum point contact. Science $\mathbf{2 8 9}$, 2323-2326 (2000).

29. Fallahi, P. et al. Imaging a single-electron quantum dot. Nano Lett. 5, 223-226 (2005).

30. Kuhl, U., Persson, E., Barth, M. \& Stöckmann, H.-J. Mixing of wavefunctions in rectangular microwave billiards. Eur. Phys. J. B 17, 253-259 (2000).

31. Gokirmak, A., Wu, D.-H., Bridgewater, J. S. A. \& Anlage, S. M. Scanned perturbation technique for imaging electromagnetic standing wave patterns of microwave cavities. Rev. Sci. Instrum. 69, 3410-3417 (1998).

32. Crampin, S. Electron states in quantum corrals. Phil. Trans. R. Soc. Lond. A 362, 1149-1161 (2004).

\section{Acknowledgements}

This work was supported by the US Office of Naval Research, the US National Science Foundation, the US Department of Energy, the Research Corporation and the Stanford-IBM Center for Probing the Nanoscale. We acknowledge the US NDSEG program (C.R.M.) and the Alfred P. Sloan Foundation

(H.C.M.) for fellowship support during this project. We thank D.-H. Lee, B. Sundaram, A. Bernevig D. Haldane and D. Eigler for discussions and W. Mar for technical assistance.

Correspondence and requests for materials should be addressed to H.C.M.

Supplementary Information accompanies this paper on www.nature.com/naturephysics. 